Regards sur l'économie allemande

Bulletin économique du CIRAC

$106 \mid 2012$

Varia

\title{
Ambivalences franco-allemandes persistantes sur l'intégration européenne
}

René Lasserre

\section{OpenEdition}

1 Journals

Édition électronique

URL : http://journals.openedition.org/rea/4461

DOI : $10.4000 /$ rea.4461

ISBN : 978-2-8218-1422-6

ISSN : 1965-0787

\section{Éditeur}

CIRAC

Édition imprimée

Date de publication : 27 octobre 2012

Pagination : 1-2

ISSN : 1156-8992

Référence électronique

René Lasserre, «Ambivalences franco-allemandes persistantes sur l'intégration européenne » Regards sur l'économie allemande [En ligne], 106 | octobre 2012, mis en ligne le 02 novembre 2012, consulté le 22 septembre 2020. URL : http://journals.openedition.org/rea/4461 ; DOI : https://doi.org/ 10.4000/rea.4461 


\section{Ambivalences franco-allemandes persistantes sur l'intégration européenne}

Après la longue période d'immobilisme qui a précédé la mise en place, il y tout juste un an, du plan de sauvegarde et de solidarité financière au sein de l'UEM, l'année qui vient de s'écouler a été marquée par une série d'avancées décisives sur la voie du sauvetage de l'Euro. C'est ainsi que le Pacte budgétaire européen adopté à la fin 2011 déboucha en mars 2012 sur l'adoption du Traité sur la stabilité, la coordination et la gouvernance (TSCG) puis sur la mise en place, après sa validation par le Tribunal constitutionnel de Karlsruhe, du Mécanisme européen de stabilité (MES). Entretemps, le sommet européen de juin, amendant le pacte de stabilité par un pacte de croissance, ouvrait la voie à une supervision et à une recapitalisation des banques pour leur permettre d'assurer le rachat de la dette souveraine. Enfin et surtout, la décision courageuse annoncée le 6 septembre par le Président de la BCE, d'intervenir sans limites pour racheter la dette publique des Etats, achevait de ramener le calme sur les marchés, mettant ainsi durablement l'Euro à l'abri des turbulences.

D'autres signaux sont venus tout récemment conforter l'embellie. La ratification du TSCG et la confirmation par la France de son engagement de retour à l'équilibre budgétaire, l'adoption de la taxe sur les transactions financières par onze Etats européens dans le sillage de la France et de l'Allemagne, ainsi que l'engagement conjoint de ces dernières d'assurer le maintien de la Grèce dans la Zone Euro semblaient augurer, après les divergences affichées au cours des premiers mois de la présidence de François Hollande, d'une normalisation progressive du "couple franco-allemand ". D'autant que de part et d'autre était conjointement réaffirmée la volonté d'avancer dans le sens de l'acceptation de règles communes, d'une cohérence et d'une intégration plus poussées des politiques budgétaires, économiques et financières au sein de la zone Euro.

Il est apparu toutefois qu'à l'approche du sommet européen des 18 et 19 octobre, la recherche conjointe de nouveaux terrains d'entente entre la France et l'Allemagne a très vite tourné court. La poursuite de nouvelles avancées sur les chantiers des politiques intégrées a cédé le pas à l'affichage réitéré de positions de principe toujours aussi peu conciliables, de sorte que le sommet a davantage donné lieu à des compromis de calendrier et de procédure qu'il n'a débouché sur des résultats concrets. Ainsi le gouvernement allemand persiste-t-il à vouloir faire prévaloir, tant aux yeux des marchés que de son opinion publique interne, un cadre de politique économique commune qui privilégie le principe de responsabilité budgétaire des Etats sur celui de la péréquation solidaire au bénéfice des pays les moins vertueux. Ce qui le conduit à plaider avec constance et fermeté pour l'adoption et le contrôle de normes financières strictes au sein de l'Union. A l'inverse, le gouvernement français, au nom du principe de solidarité communautaire avec les plus faibles, mais aussi d'une tradition de politique économique nationale qui privilégie une promotion de la croissance fondée sur la dépense publique, se montre-il réfractaire à des disciplines budgétaires communautaires contraignantes. Inversement, il se montre beaucoup plus empressé à recourir aux différentes formes de mutualisation de la dette et de péréquation financière entre les Etats pour promouvoir des politiques de relance et réduire la disparité des performances territoriales.

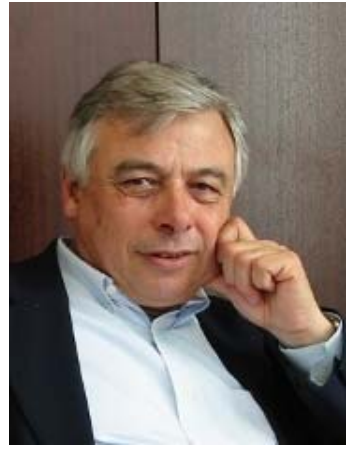

Prof. René Lasserre, Directeur du CIRAC 
Dans cette constellation habituelle, le Président français a eu beau jeu d'opposer une fin de non-recevoir à la proposition de Wolfgang Schäuble d'instaurer un droit d'intervention d'un Commissaire européen aux Affaires monétaires sur les budgets nationaux, au nom du fait que l'union budgétaire n'était pas à l'ordre du jour. Mettant ainsi un coup d'arrêt au renforcement des disciplines budgétaires, il pouvait alors s'avancer sur un autre terrain et s'ériger en promoteur de l'Union bancaire engagée lors du Sommet de juin. Soutenu par les Pays du Sud, le Président Hollande fit valoir que l'urgence appelait des avancées rapides, dès l'échéance du $1^{\mathrm{er}}$ janvier, sur la supervision bancaire afin que les banques espagnoles, italiennes ou portugaises puissent bénéficier dès que possible des concours du MES pour enrayer la flambée des taux. En cela, il entendait engager concrètement l'Union dans un processus de mutualisation des garanties bancaires, qu'il n'hésita d'ailleurs pas, dans une déclaration de presse parallèle, à considérer comme une première étape vers la mise en place ultérieure d'euroobligations.

La Chancelière n'a pas manqué d'exprimer les réserves de l'Allemagne vis-àvis du projet d'union bancaire, moins sur le plan des principes que sur celui des modalités. Elle invoqua ainsi les difficultés techniques à mettre en place, dans des délais aussi brefs, sous l'égide de la BCE, une supervision bancaire opérationnelle. De même en souligna-t-elle le caractère général et uniforme, peu compatible avec l'architecture des systèmes bancaires nationaux, et notamment avec le rôle spécifique des banques régionales allemandes. Entre les positions française et allemande, dont l'éloignement n'avait jusqu'alors jamais été aussi manifeste à l'ouverture d'un sommet, s'est néanmoins dégagé un compromis que l'on peut qualifier de conservatoire au sens où il réussit à préserver la dynamique de l'intégration économique et financière, sans toutefois générer de nouvelles et réelles convergences sur le fond.

A côté de quelques avancées symboliques destinées à concrétiser les acquis du Pacte de croissance et de la taxe sur les transactions financières, l'accord obtenu sur la définition d'un cadre d'action graduelle pour la mise en œuvre de l'union bancaire ouvre en effet une nouvelle étape importante sur la voie de l'intégration économique. D'abord au plan des objectifs, dans la mesure où il engage la mise en place d'une régulation bancaire intégrée pour le financement de l'économie réelle au sein de la zone Euro. Au plan de la méthode ensuite, au sens où il en formalise les étapes avec la définition d'un cadre juridique et la création d'un organe de surveillance comme préalables à une recapitalisation effective des banques en difficulté. Tâche de longue haleine, dont on peut d'ailleurs se demander si l'échéance prévisible d'une année ne présente pas l'avantage, de part et d'autre du Rhin, de se situer au-delà des urgences politiques immédiates.

Reste cependant la question cruciale de la consolidation budgétaire, point de divergence essentiel entre la France et l'Allemagne, où chacun campe sur ses positions, mais sur lequel visiblement le Président français, cédant à la métaphore facile de la " maison de redressement " et invoquant le spectre de la récession, persiste à vouloir repousser l'idée de la supervision budgétaire européenne à des jours meilleurs. Non sans quelque illusion, car il est à craindre que la stratégie de consolidation budgétaire qu'il a choisie, et qui consiste à tenter de réduire la dette sans s'attaquer à une réduction conséquente de la dépense, mette prochainement la France en situation difficile sur la scène européenne.

René Lasserre, le 20 octobre 2012 